TRANSACTIONS OF THE

AMERICAN MATHEMATICAL SOCIETY

Volume 361, Number 12, December 2009, Pages 6387-6405

S 0002-9947(09)04399-2

Article electronically published on June 18, 2009

\title{
ENTROPY SOLUTIONS FOR THE $p(x)$-LAPLACE EQUATION
}

\author{
MANEL SANCHÓN AND JOSÉ MIGUEL URBANO
}

\begin{abstract}
We consider a Dirichlet problem in divergence form with variable growth, modeled on the $p(x)$-Laplace equation. We obtain existence and uniqueness of an entropy solution for $L^{1}$ data, as well as integrability results for the solution and its gradient. The proofs rely crucially on a priori estimates in Marcinkiewicz spaces with variable exponent, for which we obtain new inclusion results of independent interest.
\end{abstract}

\section{INTRODUCTION}

Partial differential equations with nonlinearities involving nonconstant exponents have attracted an increasing amount of attention in recent years. Perhaps the impulse for this comes from the sound physical applications in play, or perhaps it is just the thrill of developing a mathematical theory where PDEs again meet functional analysis in a truly two-way street.

The development, mainly by Rưžička 28, of a theory modeling the behavior of electrorheological fluids, an important class of non-Newtonian fluids, seems to have boosted a still far from completed effort to study and understand nonlinear PDEs involving variable exponents. Other applications relate to image processing ( $c f$. 8] $)$, elasticity ( $c f$. 31]), the flow in porous media ( $c f$. [4] and [21]), and problems in the calculus of variations involving variational integrals with nonstandard growth ( $c f$. [31], 27], and [1]). This, in turn, gave rise to a revival of the interest in Lebesgue and Sobolev spaces with variable exponent, the origins of which can be traced back to the work of Orlicz in the 1930s. An account of recent advances, some open problems, and an extensive list of references can be found in the interesting survey by Diening et al. 14. Meanwhile, among several other contributions, the introduction by Sharapudinov 29] of the Luxemburg norm and the work of Kováčik and Rákosník 23., where many of the basic properties of these spaces are established, were crucial developments.

In this paper, we consider a problem with potential applications to the modeling of combustion, thermal explosions, nonlinear heat generation, gravitational equilibrium of polytropic stars, glaciology, non-Newtonian fluids, and the flow through

Received by the editors June 15, 2006 and, in revised form, October 10, 2007.

2000 Mathematics Subject Classification. Primary 35J70; Secondary 35D05, 35D10, $46 \mathrm{E} 35$.

The research of the first author was partially supported by CMUC/FCT and MCYT grants BMF2002-04613-C03, MTM2005-07660-C02.

The research of the second author was partially supported by CMUC/FCT and Project POCI/MAT/57546/2004. 
porous media. Many of these models have already been analyzed for constant exponents of nonlinearity ( $c f .[12$, [10, 9], 18], [30], and the references therein), but it seems to be more realistic to assume the exponent to be variable.

Let $\Omega$ be a smooth bounded domain in $\mathbb{R}^{N}$ and consider the elliptic problem

$$
\left\{\begin{aligned}
-\operatorname{div}(a(x, \nabla u)) & =f(x) & & \text { in } \quad \Omega, \\
u & =0 & & \text { on } \quad \partial \Omega,
\end{aligned}\right.
$$

where $f \in L^{1}(\Omega)$ and $a: \Omega \times \mathbb{R}^{N} \rightarrow \mathbb{R}^{N}$ is a Carathéodory function (that is, $a(\cdot, \xi)$ is measurable in $\Omega$, for every $\xi \in \mathbb{R}^{N}$, and $a(x, \cdot)$ is continuous in $\mathbb{R}^{N}$, for almost every $x \in \Omega$ ), such that the following assumptions hold:

$$
a(x, \xi) \cdot \xi \geq b|\xi|^{p(x)},
$$

for almost every $x \in \Omega$ and for every $\xi \in \mathbb{R}^{N}$, where $b$ is a positive constant;

$$
|a(x, \xi)| \leq \beta\left(j(x)+|\xi|^{p(x)-1}\right),
$$

for almost every $x \in \Omega$ and for every $\xi \in \mathbb{R}^{N}$, where $j$ is a nonnegative function in $L^{p^{\prime}(\cdot)}(\Omega)$ and $\beta>0$

$$
\left(a(x, \xi)-a\left(x, \xi^{\prime}\right)\right) \cdot\left(\xi-\xi^{\prime}\right)>0,
$$

for almost every $x \in \Omega$ and for every $\xi, \xi^{\prime} \in \mathbb{R}^{N}$, with $\xi \neq \xi^{\prime}$.

Hypotheses (1.2)-(1.4) are the natural extensions of the classical assumptions in the study of nonlinear monotone operators in divergence form for constant $p(\cdot) \equiv p$ (cf. 26]).

Concerning the exponent $p(\cdot)$ appearing in (1.2) and (1.3), we assume it is a measurable function $p(\cdot): \Omega \rightarrow \mathbb{R}$ such that

$$
\left\{\begin{array}{l}
\exists C>0:|p(x)-p(y)| \leq \frac{C}{-\ln |x-y|}, \quad \text { for }|x-y|<\frac{1}{2} \\
1<\underset{x \in \Omega}{\operatorname{ess} \inf _{x \in \Omega}} p(x) \leq \underset{x \in}{\operatorname{ess} \sup } p(x)<N .
\end{array}\right.
$$

The first condition says that $p(\cdot)$ belongs to the class of log-Hölder continuous functions. These assumptions allow us, in particular, to exploit the functional analytical properties of Lebesgue and Sobolev spaces with variable exponent (see section 2) arising in the study of problem (1.1).

A weak solution of (1.1) is a function $u \in W_{0}^{1,1}(\Omega)$ such that $a(\cdot, \nabla u) \in L_{\mathrm{loc}}^{1}(\Omega)$ and

$$
\int_{\Omega} a(x, \nabla u) \cdot \nabla \varphi d x=\int_{\Omega} f(x) \varphi d x, \quad \text { for all } \varphi \in C_{0}^{\infty}(\Omega) .
$$

A weak energy solution is a weak solution such that $u \in W_{0}^{1, p(\cdot)}(\Omega)$.

The model case for (1.1) is the Dirichlet problem for the $p(x)$-Laplacian operator $\Delta_{p(x)} u:=\operatorname{div}\left(|\nabla u|^{p(x)-2} \nabla u\right)$,

$$
\left\{\begin{aligned}
-\Delta_{p(x)} u & =f(x) & & \text { in } \quad \Omega, \\
u & =0 & & \text { on }
\end{aligned}\right.
$$

This and other related problems (where $f$ is replaced by a nonlinear function depending on $u$ ) have been studied recently in several papers ( $c f$., for example, [16] for existence and uniqueness or [17] for Hölder continuity) in the framework of weak energy solutions. These results require the assumption that the right-hand side $f$ has enough integrability. 
Assuming that $f$ is merely in $L^{1}(\Omega)$, we need to work with entropy solutions, which are required to be less regular than weak solutions. The notion of entropy solution was introduced by Bénilan et al. [5] for problem (1.1) in the framework of a constant $p(\cdot) \equiv p$, and existence and uniqueness were established, together with some estimates for the solution and its weak gradient. Using essentially the same tools, Alvino et al. 3] proved existence of an entropy solution for elliptic problems with degenerate coercivity, still in the context of constant exponents.

The main purpose of this paper is to extend the results in [5] to a nonconstant $p(\cdot)$. Defining the truncation function $T_{t}$ by

$$
T_{t}(s):=\max \{-t, \min \{t, s\}\}, \quad s \in \mathbb{R},
$$

we start by extending the notion of entropy solution to problem (1.1) as follows:

Definition 1.1. A measurable function $u$ is an entropy solution to problem (1.1) if, for every $t>0, T_{t}(u) \in W_{0}^{1, p(\cdot)}(\Omega)$ and

$$
\int_{\Omega} a(x, \nabla u) \cdot \nabla T_{t}(u-\varphi) d x \leq \int_{\Omega} f(x) T_{t}(u-\varphi) d x
$$

for all $\varphi \in W_{0}^{1, p(\cdot)}(\Omega) \cap L^{\infty}(\Omega)$.

A function $u$ such that $T_{t}(u) \in W_{0}^{1, p(\cdot)}(\Omega)$, for all $t>0$, does not necessarily belong to $W_{0}^{1,1}(\Omega)$. However, it is possible to define its weak gradient (see Proposition 3.1 below), still denoted by $\nabla u$.

Let us introduce the following notation:

$$
p_{-}:=\underset{x \in \Omega}{\operatorname{ess} \inf } p(x) \quad \text { and } \quad p_{+}:=\underset{x \in \Omega}{\operatorname{ess} \sup } p(x) ;
$$

given two bounded measurable functions $p(\cdot), q(\cdot): \Omega \rightarrow \mathbb{R}$, we write

$$
q(\cdot) \ll p(\cdot) \quad \text { if }(p-q)_{-}>0 .
$$

Assuming (1.5), the critical Sobolev exponent and the conjugate of $p(\cdot)$ are, respectively,

Our main result is

$$
p^{*}(\cdot)=\frac{N p(\cdot)}{N-p(\cdot)} \quad \text { and } \quad p^{\prime}(\cdot)=\frac{p(\cdot)}{p(\cdot)-1} .
$$

Theorem 1.2. Assume (1.2) - (1.5) and $f \in L^{1}(\Omega)$. There exists a unique entropy solution $u$ to problem (1.1). Moreover, $|u|^{q(\cdot)} \in L^{1}(\Omega)$, for all $0 \ll q(\cdot) \ll q_{0}(\cdot)$, and $|\nabla u|^{q(\cdot)} \in L^{1}(\Omega)$, for all $0 \ll q(\cdot) \ll q_{1}(\cdot)$, where

$$
q_{0}(\cdot):=\frac{p^{*}(\cdot)}{p_{+}^{\prime}} \quad \text { and } \quad q_{1}(\cdot):=\frac{q_{0}(\cdot)}{q_{0}(\cdot)+1} p(\cdot) .
$$

The proof of this result will be decomposed into several steps. First, we obtain $a$ priori estimates for entropy solutions in Marcinkiewicz spaces with variable exponent. Despite the fact that the theory of functional spaces with variable exponent is developing quickly, the extension of classical Marcinkiewicz spaces is, to the best of our knowledge, undertaken here for the first time. From these estimates, we derive uniform bounds in Lebesgue spaces of variable exponent for an entropy solution and its weak gradient (see Corollaries 3.5 and 3.7 in section 3). The uniqueness follows from choosing adequate test functions in the entropy condition (1.8) and 
using the a priori estimates. Finally, the existence is obtained by passing to the limit in a sequence of weak energy solutions of adequate approximated problems.

Our other theorem concerns weak solutions and extends the results obtained by Boccardo and Gallouët [6, 7] in the context of a constant $p(\cdot) \equiv p$.

Theorem 1.3. Assume (1.2) (1.5) and $f \in L^{1}(\Omega)$. Let $q_{0}(\cdot), q_{1}(\cdot)$ be given by (1.9), and let $u$ be the entropy solution of (1.1). If $2-1 / N \ll p(\cdot)$, then $u \in$ $L^{q(\cdot)}(\Omega)$, for all $1 \ll q(\cdot) \ll q_{0}(\cdot)$, and $u \in W_{0}^{1, q(\cdot)}(\Omega)$, for all $1 \ll q(\cdot) \ll q_{1}(\cdot)$. If, in addition, $p(\cdot)-1 \ll q_{1}(\cdot)$, then $u$ is a weak solution of (1.1).

We will see later that $1 \ll q_{1}(\cdot)$ if and only if $2-1 / N \ll p(\cdot)$, and hence, by Theorem 1.2, the entropy solution $u$ belongs to $W_{0}^{1,1}(\Omega)$ if $2-1 / N \ll p(\cdot)$. We also remark that, in the constant case, we have

$$
q_{0}=\frac{N(p-1)}{N-p} \quad \text { and } \quad q_{1}=\frac{N(p-1)}{N-1},
$$

which coincide with the exponents in $\left[5\right.$. The additional assumption $p(\cdot)-1 \ll q_{1}(\cdot)$ is needed to show that the entropy solution is indeed a weak solution, i.e., that it satisfies the equation in the distributional sense. Later, we discuss in detail the significance of this assumption and conclude, in particular, that it is not stringent up to dimension $N=10$ (see Remark 5.7).

In this paper, we always assume that $f \in L^{1}(\Omega)$; increasing the integrability of $f$, one expects to obtain more regularity but, for variable exponents, most results in this direction are still missing.

A few comments about known regularity results for the constant exponent case, in terms of the integrability of the right-hand side $f$, are in order. Assume $p(\cdot) \equiv p$ is constant and the right-hand side $f \in L^{m}(\Omega)$, for some $m \geq 1$. The existence and uniqueness of an entropy solution $u$ of problem (1.1) is obtained in [5]. Define the numbers

$$
\bar{m}:=\frac{N}{N(p-1)+1} \quad \text { and } \quad \tilde{m}:=\left(p^{*}\right)^{\prime}=\frac{N p}{N(p-1)+p},
$$

where $p^{*}=N p /(N-p)$ is the Sobolev exponent. The following assertions hold:

(A1) If $1 \leq m \leq \max (1, \bar{m})$, then the entropy solution $u$ satisfies $|u|^{q} \in L^{1}(\Omega)$, for all $0<q<q_{0}$, and $|\nabla u|^{q} \in L^{1}(\Omega)$, for all $0<q<q_{1}$, where

$$
q_{0}:=\frac{N m(p-1)}{N-m p} \quad \text { and } \quad q_{1}:=\frac{N m(p-1)}{N-m}
$$

(note that, when $m=1$, these numbers coincide with the ones defined in (1.9), since we are assuming that $p(\cdot) \equiv p$ is constant).

(A2) If $\max (1, \bar{m})<m<\tilde{m}$, then $u$ is a weak solution and $u \in W_{0}^{1, q_{1}}(\Omega)$ (note that $\left.q_{1}>1\right)$.

(A3) If $\tilde{m} \leq m \leq N / p$, then $u$ is a weak energy solution and $u \in W_{0}^{1, q_{1}}(\Omega)$ (note that $\left.q_{1} \geq p\right)$.

(A4) If $m>N / p$, then $u$ is a bounded weak energy solution.

The first and last assertions are proved by Alvino et al. [3]. The second one follows from the results of Boccardo and Gallouët [6, 7, and the third is a consequence of a result by Kinnunen and Zhou [22, Thm. 1.6]. It is also known that if $m>N p^{\prime}$, then $u \in C_{\text {loc }}^{1, \alpha}(\Omega)$, a result due to DiBenedetto [10]. 
For a variable exponent $p(\cdot)$ much less is known. If $f \in W^{-1, p^{\prime}(\cdot)}(\Omega)$ or, in particular, if $f \in L^{\tilde{m}(\cdot)}(\Omega)$, where $\tilde{m}(\cdot):=\left(p(\cdot)^{*}\right)^{\prime}$, the existence and uniqueness of a weak energy solution to problem (1.1) is a straightforward generalization of the results obtained by Fan and Zhang [16] for the model problem (1.7).

Recently, Acerbi and Mingione [2] derived Calderón-Zygmund type estimates for (1.1), extending previous results of DiBenedetto and Manfredi [11 for the model problem (1.7) and $p(\cdot) \equiv p$ constant. Using their estimates it is easy to prove the following result.

Proposition 1.4. Assume (1.2) -(1.5) and $f \in L_{\mathrm{loc}}^{m(\cdot)}(\Omega)$, where

$$
m(\cdot):=\frac{N p(\cdot) q}{N(p(\cdot)-1)+p(\cdot) q} \quad \text { with } \quad q \geq 1 .
$$

The unique weak energy solution $u$ of (1.1) satisfies $|\nabla u|^{p(\cdot)} \in L_{\mathrm{loc}}^{q}(\Omega)$.

We note that the function $m(\cdot)$ defined in (1.10) satisfies

$$
\tilde{m}(\cdot)<m(\cdot)<N, \quad \text { for all } q>1 \text {. }
$$

As an immediate consequence, one obtains $u \in W_{\text {loc }}^{1, r(\cdot)}(\Omega)$, for all $r(\cdot) \in L^{\infty}(\Omega)$, if $f \in L_{\text {loc }}^{N}(\Omega)$. We note that, in the case of constant exponents, Proposition 1.4 states that for $f \in L_{\text {loc }}^{m}(\Omega)$, with $m \geq \tilde{m}$, we have $u \in W_{\text {loc }}^{1, q_{1}}(\Omega)$. Moreover, as a consequence of Sobolev embedding, it follows that $u \in C_{\mathrm{loc}}^{0, \alpha}(\Omega)$ if $m>N / p$. We thus recover local versions of assertions (A3) and (A4). Therefore, to obtain (A3) and (A4) using this reasoning, it would be necessary to prove a global version of Proposition 1.4 for a nonconstant $q(\cdot)$.

Finally, since Theorem 1.2 guarantees the existence and uniqueness of an entropy solution for (1.1), the extension of (A1) and (A2) for variable exponents only requires a priori estimates for such a solution. We feel that the techniques needed to obtain such estimates are slight modifications of the ones used in section 3 in the $L^{1}$ case but this extension remains open.

The paper is organized as follows. In section 2, we recall the definitions of Lebesgue and Sobolev spaces with variable exponent and some of their properties. Then, we introduce Marcinkiewicz spaces with variable exponent and establish their relation with Lebesgue spaces. In section 3, we obtain a priori estimates for an entropy solution and its weak gradient. In section 4 we prove uniqueness of entropy solutions. Finally, in section 5] we consider approximate problems and, using the $a$ priori estimates, we establish the existence results.

\section{MARCINKIEWICZ SPACES WITH VARIABLE EXPONENT}

In this section, we define Marcinkiewicz spaces with variable exponent and investigate their relation with Lebesgue spaces. To the best of our knowledge, this definition is considered here for the first time and the properties obtained are new.

We start with a brief overview of the state of the art concerning Lebesgue spaces with variable exponent, and Sobolev spaces modeled upon them. We define the Lebesgue space with variable exponent $L^{p(\cdot)}(\Omega)$ as the set of all measurable functions $u: \Omega \rightarrow \mathbb{R}$ for which the convex modular

$$
\varrho_{p(\cdot)}(u)=\int_{\Omega}|u|^{p(x)} d x
$$


is finite. If the exponent is bounded, i.e., if $p_{+}<\infty$, then the expression

$$
\|u\|_{p(\cdot)}:=\inf \left\{\lambda>0: \varrho_{p(\cdot)}(u / \lambda) \leq 1\right\}
$$

defines a norm in $L^{p(\cdot)}(\Omega)$, called the Luxemburg norm. One central property of $L^{p(\cdot)}(\Omega)$ is that the norm and the modular topologies coincide; i.e., $\varrho_{p(\cdot)}\left(u_{n}\right) \rightarrow 0$ if and only if $\left\|u_{n}\right\|_{p(\cdot)} \rightarrow 0$. The space $\left(L^{p(\cdot)}(\Omega),\|\cdot\|_{p(\cdot)}\right)$ is a separable Banach space. Moreover, if $p_{-}>1$, then $L^{p(\cdot)}(\Omega)$ is uniformly convex, hence reflexive, and its dual space is isomorphic to $L^{p^{\prime}(\cdot)}(\Omega)$, where $1 / p(x)+1 / p^{\prime}(x)=1$. Finally, we have the Hölder inequality:

$$
\left|\int_{\Omega} u v d x\right| \leq\left(\frac{1}{p_{-}}+\frac{1}{p_{-}^{\prime}}\right)\|u\|_{p(\cdot)}\|v\|_{p^{\prime}(\cdot)},
$$

for all $u \in L^{p(\cdot)}(\Omega)$ and $v \in L^{p^{\prime}(\cdot)}(\Omega)$.

Now, let

$$
W^{1, p(\cdot)}(\Omega):=\left\{u \in L^{p(\cdot)}(\Omega):|\nabla u| \in L^{p(\cdot)}(\Omega)\right\},
$$

which is a Banach space equipped with the norm

$$
\|u\|_{1, p(\cdot)}:=\|u\|_{p(\cdot)}+\|\nabla u\|_{p(\cdot)} .
$$

By $W_{0}^{1, p(\cdot)}(\Omega)$ we denote the closure of $C_{0}^{\infty}(\Omega)$ in $W^{1, p(\cdot)}(\Omega)$.

The proofs of the following two results can be found in [20] and [13], respectively.

Proposition 2.1 (The $p(\cdot)$-Poincaré inequality). Let $\Omega$ be a bounded open set and let $p(\cdot): \Omega \rightarrow[1, \infty)$ satisfy (1.5). There exists a constant $C$, depending only on $p(\cdot)$ and $\Omega$, such that the inequality

$$
\|u\|_{p(\cdot)} \leq C\|\nabla u\|_{p(\cdot)}
$$

holds for every $u \in W_{0}^{1, p(\cdot)}(\Omega)$.

Proposition 2.2 (Sobolev embedding). Let $\Omega$ be a bounded open set, with a Lipschitz boundary, and let $p(\cdot): \Omega \rightarrow[1, \infty)$ satisfy (1.5). Then we have the following continuous embedding:

$$
W^{1, p(\cdot)}(\Omega) \hookrightarrow L^{p^{*}(\cdot)}(\Omega)
$$

where $p^{*}(\cdot)=\frac{N p(\cdot)}{N-p(\cdot)}$.

Now, we give a useful result in order to apply the Sobolev inequality ( $c f$. [15]).

Lemma 2.3. Let $p(\cdot)$ and $q(\cdot)$ be measurable functions such that $p(\cdot) \in L^{\infty}(\Omega)$ and $1 \leq p(x) q(x) \leq+\infty$, for a.e. $x \in \Omega$. Let $f \in L^{q(\cdot)}(\Omega), f \not \equiv 0$. Then

$$
\begin{aligned}
\|f\|_{p(\cdot) q(\cdot)}^{p_{+}} \leq\left\||f|^{p(\cdot)}\right\|_{q(\cdot)} \leq\|f\|_{p(\cdot) q(\cdot)}^{p_{-}} & \text {if }\|f\|_{p(\cdot) q(\cdot)} \leq 1, \\
\|f\|_{p(\cdot) q(\cdot)}^{p_{-}} \leq\left\||f|^{p(\cdot)}\right\|_{q(\cdot)} \leq\|f\|_{p(\cdot) q(\cdot)}^{p_{+}} & \text {if }\|f\|_{p(\cdot) q(\cdot)} \geq 1 .
\end{aligned}
$$

In particular, if $p(\cdot) \equiv p$ is constant, then

$$
\left\||f|^{p}\right\|_{q(\cdot)}=\|f\|_{p q(\cdot)}^{p} .
$$

This closes our brief tour of Lebesgue and Sobolev spaces with variable exponent. Let's now consider Marcinkiewicz spaces with variable exponent. To the best of our knowledge, the next definition is new. 
Definition 2.4. Let $q(\cdot)$ be a measurable function such that $q_{-}>0$. We say that a measurable function $u$ belongs to the Marcinkiewicz space $M^{q(\cdot)}(\Omega)$ if there exists a positive constant $M$ such that

$$
\int_{\{|u|>t\}} t^{q(x)} d x \leq M, \text { for all } t>0 .
$$

We remark that for $q(\cdot) \equiv q$ constant this definition coincides with the classical definition of the Marcinkiewicz space $M^{q}(\Omega)(c f$. 25]). Moreover, it is clear that $u \in M^{q(\cdot)}(\Omega)$ if $|u|^{q(\cdot)} \in L^{1}(\Omega)$. Indeed,

$$
\int_{\{|u|>t\}} t^{q(x)} d x \leq \int_{\Omega}|u|^{q(x)} d x, \quad \text { for all } t>0 .
$$

In particular, $L^{q(\cdot)}(\Omega) \subset M^{q(\cdot)}(\Omega)$, for all $q(\cdot) \geq 1$.

For constant exponents it is straightforward to prove some sort of reciproque: if $u \in M^{r}(\Omega)$, then $|u|^{q} \in L^{1}(\Omega)$, for all $0<q<r$. The following result extends this assertion to the nonconstant setting; unlike the constant case, the proof presents some difficulties.

Proposition 2.5. Let $r(\cdot)$ and $q(\cdot)$ be bounded functions such that $0 \ll q(\cdot) \ll r(\cdot)$ and let $\epsilon:=(r-q)_{-}>0$. If $u \in M^{r(\cdot)}(\Omega)$, then

$$
\int_{\Omega}|u|^{q(x)} d x \leq 2|\Omega|+\left(r_{+}-\epsilon\right) \frac{M}{\epsilon},
$$

where $M$ is the constant appearing in the definition of $M^{r(\cdot)}(\Omega)$. In particular, $M^{r(\cdot)}(\Omega) \subset L^{q(\cdot)}(\Omega)$, for all $1 \leq q(\cdot) \ll r(\cdot)$.

Proof. Noting that $0 \ll q(\cdot) \leq r(\cdot)-\epsilon$, we define the a.e. differentiable function

$$
\varphi(t):=\int_{\{|u|>t\}} t^{r(x)-\epsilon} d x, \text { for all } t>0 .
$$

Writing its derivative as

$$
\varphi^{\prime}(t)=\int_{\{|u|>t\}}(r(x)-\epsilon) t^{r(x)-\epsilon-1} d x-\lim _{h \downarrow 0} \frac{1}{h} \int_{\{t-h<|u| \leq t\}} t^{r(x)-\epsilon} d x,
$$

we obtain

$$
\begin{aligned}
-\frac{d}{d t} \int_{\{|u|>t\}}|u|^{r(x)-\epsilon} d x & =\lim _{h \downarrow 0} \frac{1}{h} \int_{\{t-h<|u| \leq t\}}|u|^{r(x)-\epsilon} d x \\
& \leq \lim _{h \downarrow 0} \frac{1}{h} \int_{\{t-h<|u| \leq t\}} t^{r(x)-\epsilon} d x \\
& =\int_{\{|u|>t\}}(r(x)-\epsilon) t^{r(x)-\epsilon-1} d x-\varphi^{\prime}(t) .
\end{aligned}
$$


Using the previous inequality and remarking that $0 \leq \varphi(t) \leq M / t^{\epsilon}$, for all $t>0$, since $u \in M^{r(\cdot)}(\Omega)$, we derive the estimate

$$
\begin{aligned}
\int_{\Omega}|u|^{q(x)} d x & \leq|\Omega|+\int_{\{|u|>1\}}|u|^{r(x)-\epsilon} d x \\
& =|\Omega|+\int_{1}^{\infty}\left(-\frac{d}{d t} \int_{\{|u|>t\}}|u|^{r(x)-\epsilon} d x\right) d t \\
& \leq|\Omega|+\int_{1}^{\infty}\left(\int_{\{|u|>t\}}(r(x)-\epsilon) t^{r(x)-\epsilon-1} d x-\varphi^{\prime}(t)\right) d t \\
& \leq|\Omega|+\left(r^{+}-\epsilon\right) \int_{1}^{\infty} \frac{1}{t^{\epsilon+1}}\left(\int_{\{|u|>t\}} t^{r(x)} d x\right) d t+\varphi(1) \\
& \leq 2|\Omega|+\left(r^{+}-\epsilon\right) \int_{1}^{\infty} \frac{M}{t^{\epsilon+1}} d t \\
& =2|\Omega|+\left(r^{+}-\epsilon\right) \frac{M}{\epsilon}
\end{aligned}
$$

and the result follows.

\section{A PRIORI ESTIMATES}

We start with the existence of the weak gradient for every measurable function $u$ such that $T_{t}(u) \in W_{0}^{1, p(\cdot)}(\Omega)$, for all $t>0$.

Proposition 3.1. If $u$ is a measurable function such that $T_{t}(u) \in W_{0}^{1, p(\cdot)}(\Omega)$, for all $t>0$, then there exists a unique measurable function $v: \Omega \rightarrow \mathbb{R}^{N}$ such that

$$
v \chi_{\{|u|<t\}}=\nabla T_{t}(u) \text { for a.e. } x \in \Omega \text {, and for all } t>0,
$$

where $\chi_{E}$ denotes the characteristic function of a measurable set $E$. Moreover, if $u$ belongs to $W_{0}^{1,1}(\Omega)$, then $v$ coincides with the standard distributional gradient of $u$.

Proof. The result follows from [3, Theorem 1.5], since

$$
T_{t}(u) \in W_{0}^{1, p(\cdot)}(\Omega) \subset W_{0}^{1, p_{-}}(\Omega), \quad \text { for all } t>0 .
$$

The next result provides estimates in Marcinkiewicz spaces (and hence, by Proposition 2.5, in Lebesgue spaces) for an entropy solution of (1.1).

Proposition 3.2. Assume (1.2)-(1.5) and $f \in L^{1}(\Omega)$. If $u$ is an entropy solution of (1.1), then there exists a positive constant $M$, depending only on $p(\cdot), N$, and $\Omega$, such that

$$
\int_{\{|u|>t\}} t^{p^{*}(x) / p_{+}^{\prime}} d x \leq M\left(\frac{\|f\|_{1}}{b}+1\right)^{p_{+}^{*} / p_{-}}, \quad \text { for all } t>0 .
$$

Proof. Taking $\varphi=0$ in the entropy inequality (1.8) and using (1.2), we obtain

$$
\begin{aligned}
b \int_{\Omega}\left|\nabla T_{t}(u)\right|^{p(x)} d x & \leq \int_{\{|u| \leq t\}} a(x, \nabla u) \cdot \nabla u d x \\
& \leq \int_{\Omega} f(x) T_{t}(u) d x \leq t\|f\|_{1},
\end{aligned}
$$


for all $t>0$. Therefore, defining $\psi:=T_{t}(u) / t$, we have, for all $t>0$,

$$
\int_{\Omega} t^{p(x)-1}|\nabla \psi|^{p(x)} d x=\frac{1}{t} \int_{\Omega}\left|\nabla T_{t}(u)\right|^{p(x)} d x \leq M_{1}:=\frac{\|f\|_{1}}{b} .
$$

Let $\gamma>0$ be a number to be chosen later. Using the Sobolev inequality (2.3) and Lemma 2.3, we estimate

$$
\begin{aligned}
\int_{\{|u|>t\}} t^{p^{*}(x) / \gamma} d x & =\int_{\{|\psi|=1\}} t^{p^{*}(x) / \gamma}|\psi|^{p^{*}(x)} d x \\
& \leq \int_{\Omega}\left(t^{1 / \gamma}|\psi|\right)^{p^{*}(x)} d x \\
& \leq\left\|t^{1 / \gamma} \psi\right\|_{p^{*}(\cdot)}^{\alpha_{1}} \\
& \leq C^{\alpha_{1}}\left\|\nabla\left(t^{1 / \gamma} \psi\right)\right\|_{p(\cdot)}^{\alpha_{1}} \\
& \leq C^{\alpha_{1}}\left(\int_{\Omega}\left|\nabla\left(t^{1 / \gamma} \psi\right)\right|^{p(x)} d x\right)^{\alpha_{1} / \beta_{1}} \\
& =C^{\alpha_{1}}\left(\int_{\Omega} t^{p(x) / \gamma+1-p(x)} \frac{1}{t}\left|\nabla T_{t}(u)\right|^{p(x)} d x\right)^{\alpha_{1} / \beta_{1}},
\end{aligned}
$$

where

$$
\alpha_{1}=\left\{\begin{array}{ll}
p_{+}^{*} & \text { if } \quad\left\|t^{1 / \gamma} \psi\right\|_{p^{*}(\cdot)} \geq 1 \\
p_{-}^{*} & \text { if } \quad\left\|t^{1 / \gamma} \psi\right\|_{p^{*}(\cdot)} \leq 1
\end{array} \text { and } \beta_{1}=\left\{\begin{array}{lll}
p_{-} & \text {if } \quad\left\|\nabla\left(t^{1 / \gamma} \psi\right)\right\|_{p(\cdot)} \geq 1 \\
p_{+} & \text {if } \quad\left\|\nabla\left(t^{1 / \gamma} \psi\right)\right\|_{p(\cdot)} \leq 1 .
\end{array}\right.\right.
$$

Now, choosing $\gamma=p_{+}^{\prime}$ in (3.2), noting that $t^{p(x) / \gamma+1-p(x)} \leq 1$ for all $t \geq 1$, and using (3.1), we obtain

$$
\int_{\{|u|>t\}} t^{p^{*}(x) / p_{+}^{\prime}} d x \leq C^{\alpha_{1}}\left(M_{1}+1\right)^{p_{+}^{*} / p_{-}}
$$

for all $t \geq 1$. Finally, for $0<t<1$, we have

$$
\int_{\{|u|>t\}} t^{p^{*}(x) / p_{+}^{\prime}} d x \leq|\Omega| .
$$

Combining both estimates, the result follows.

Remark 3.3. Recalling from (1.9) that

$$
q_{0}(\cdot)=\frac{p^{*}(\cdot)}{p_{+}^{\prime}}
$$

Proposition 3.2 yields $u \in M^{q_{0}(\cdot)}(\Omega)$. We note that for $p(\cdot) \equiv p$ we have that $u \in M^{q_{0}}(\Omega)$, with

$$
q_{0}=\frac{N(p-1)}{N-p}=\frac{p^{*}}{p^{\prime}}
$$

recovering the result obtained in [5]. For the nonconstant case, it remains an open problem to show that $u \in M^{q(\cdot)}(\Omega)$, with $q(\cdot)=p^{*}(\cdot) / p^{\prime}(\cdot)$.

Remark 3.4. We stress that the dependence of the constant $M$ on $p(\cdot)$ occurs solely through the constants $p_{-}$and $p_{+}$. 
As a consequence of Proposition 2.5 and Proposition 3.2 we obtain the following result.

Corollary 3.5. Assume (1.2) -1.5$)$ and $f \in L^{1}(\Omega)$. Let

$$
q_{0}(\cdot)=\frac{p^{*}(\cdot)}{p_{+}^{\prime}} .
$$

If $u$ is an entropy solution to problem (1.1), then $|u|^{q(\cdot)} \in L^{1}(\Omega)$, for all $q(\cdot)$ such that $0 \ll q(\cdot) \ll q_{0}(\cdot)$. Moreover, there exists a constant $M_{0}$, depending only on $p(\cdot), q(\cdot), N$ and $\Omega$, such that

$$
\int_{\Omega}|u|^{q(x)} d x \leq 2|\Omega|+M_{0}\left(\frac{\|f\|_{1}}{b}+1\right)^{p_{+}^{*} / p_{-}} .
$$

Proof. Let $0 \ll q(\cdot) \ll q_{0}(\cdot)$ and define $\delta:=\left(q_{0}-q\right)_{-}>0$. By Proposition 3.2 ,

$$
\int_{\{|u|>t\}} t^{q_{0}(x)} d x \leq M\left(\frac{\|f\|_{1}}{b}+1\right)^{p_{+}^{*} / p_{-}}, \quad \text { for all } t>0,
$$

where $M$ is a positive constant, depending only on $p(\cdot), N$ and $\Omega$. From Proposition 2.5, we have

$$
\int_{\Omega}|u|^{q(x)} d x \leq 2|\Omega|+\left(q_{0}-\delta\right)_{+} \frac{M}{\delta}\left(\frac{\|f\|_{1}}{b}+1\right)^{p_{+}^{*} / p_{-}}
$$

estimate (3.4) now follows with

$$
M_{0}=\left(q_{0}-\delta\right)_{+} \frac{M}{\delta}
$$

Now, we prove a priori estimates in Marcinkiewicz spaces for the weak gradient of an entropy solution.

Proposition 3.6. Assume (1.2)-(1.5) and $f \in L^{1}(\Omega)$. Let $u$ be an entropy solution of (1.1). If there exists a positive constant $M$ such that

$$
\int_{\{|u|>t\}} t^{q(x)} d x \leq M, \quad \text { for all } t>0,
$$

then $|\nabla u|^{\alpha(\cdot)} \in M^{q(\cdot)}(\Omega)$, where $\alpha(\cdot)=p(\cdot) /(q(\cdot)+1)$. Moreover,

$$
\int_{\left\{|\nabla u|^{\alpha(\cdot)}>t\right\}} t^{q(x)} d x \leq \frac{\|f\|_{1}}{b}+M, \quad \text { for all } t>0 .
$$

Proof. Using (3.5), the definition of $\alpha(\cdot)$, and (3.1), which still holds in this setting, we have

$$
\begin{aligned}
\int_{\left\{|\nabla u|^{\alpha(x)}>t\right\}} t^{q(x)} d x & \leq \int_{\left\{|\nabla u|^{\alpha(x)}>t\right\} \cap\{|u| \leq t\}} t^{q(x)} d x+\int_{\{|u|>t\}} t^{q(x)} d x \\
& \leq \int_{\{|u| \leq t\}} t^{q(x)}\left(\frac{|\nabla u|^{\alpha(x)}}{t}\right)^{p(x) / \alpha(x)} d x+M \\
& =\frac{1}{t} \int_{\{|u| \leq t\}}\left|\nabla T_{t}(u)\right|^{p(x)} d x+M \\
& \leq \frac{\|f\|_{1}}{b}+M, \text { for all } t>0 .
\end{aligned}
$$


As a consequence of Proposition 2.5, Proposition 3.2, and Proposition 3.6, we obtain the following result.

Corollary 3.7. Assume (1.2) (1.5) and $f \in L^{1}(\Omega)$. Let $q_{0}(\cdot)$ be defined in (3.3) and let

$$
q_{1}(\cdot)=\frac{q_{0}(\cdot)}{q_{0}(\cdot)+1} p(\cdot) .
$$

If $u$ is an entropy solution of problem (1.1), then $|\nabla u|^{q(\cdot)} \in L^{1}(\Omega)$, for all $q(\cdot)$ such that $0 \ll q(\cdot) \ll q_{1}(\cdot)$. Moreover, there exist constants $M_{2}$ and $M_{3}$, depending only on $p(\cdot), q(\cdot), N$ and $\Omega$, such that

$$
\int_{\Omega}|\nabla u|^{q(x)} d x \leq 2|\Omega|+M_{2} \frac{\|f\|_{1}}{b}+M_{3}\left(\frac{\|f\|_{1}}{b}+1\right)^{p_{+}^{*} / p_{-}} .
$$

Proof. By Proposition 3.6 (and using also Proposition 3.2), we have

$$
|\nabla u|^{\alpha(\cdot)} \in M^{q_{0}(\cdot)}(\Omega), \quad \text { with } \quad \alpha(\cdot)=p(\cdot) /\left(q_{0}(\cdot)+1\right),
$$

and

$$
\int_{\left\{|\nabla u|^{\alpha(\cdot)}>t\right\}} t^{q_{0}(x)} d x \leq \frac{\|f\|_{1}}{b}+M\left(\frac{\|f\|_{1}}{b}+1\right)^{p_{+}^{*} / p_{-}}, \quad \text { for all } t>0,
$$

where $M$ is a positive constant, depending only on $p(\cdot), N$ and $\Omega$.

Let $0 \ll q(\cdot) \ll q_{1}(\cdot)$, note that $r(\cdot):=q(\cdot) / \alpha(\cdot) \ll q_{0}(\cdot)$ and define $\varrho:=$ $\left(q_{0}-r\right)_{-}>0$. From Proposition 2.5 applied to $|\nabla u|^{\alpha(\cdot)}$, we have

$$
\begin{aligned}
\int_{\Omega}|\nabla u|^{q(x)} d x & =\int_{\Omega}|\nabla u|^{\alpha(x) r(x)} d x \\
& \leq 2|\Omega|+\frac{\left(q_{0}-\varrho\right)_{+}}{\varrho}\left[\frac{\|f\|_{1}}{b}+M\left(\frac{\|f\|_{1}}{b}+1\right)^{p_{+}^{*} / p_{-}}\right],
\end{aligned}
$$

and the result follows with

$$
M_{2}=\frac{\left(q_{0}-\varrho\right)_{+}}{\varrho} \text { and } \quad M_{3}=M M_{2} .
$$

\section{UNIQUENESS OF ENTROPY SOLUTIONS}

In this section we establish the uniqueness of an entropy solution, extending the result obtained in [5] for a constant exponent.

Theorem 4.1. Assume (1.2) (1.5) and $f \in L^{1}(\Omega)$. If $u$ and $v$ are entropy solutions of (1.1), then $u=v$, a.e. in $\Omega$.

Proof. Let $h>0$. We write the entropy inequality (1.8) corresponding to the solution $u$, with $T_{h} v$ as test function, and to the solution $v$, with $T_{h} u$ as test function. Upon addition, we get

$$
\begin{gathered}
\int_{\left\{\left|u-T_{h} v\right| \leq t\right\}} a(x, \nabla u) \cdot \nabla\left(u-T_{h} v\right) d x+\int_{\left\{\left|v-T_{h} u\right| \leq t\right\}} a(x, \nabla v) \cdot \nabla\left(v-T_{h} u\right) d x \\
\leq \int_{\Omega} f(x)\left(T_{t}\left(u-T_{h} v\right)+T_{t}\left(v-T_{h} u\right)\right) d x .
\end{gathered}
$$


Define

$$
\begin{gathered}
E_{1}:=\{|u-v| \leq t,|v| \leq h\}, \\
E_{2}:=E_{1} \cap\{|u| \leq h\}, \quad \text { and } \quad E_{3}:=E_{1} \cap\{|u|>h\} .
\end{gathered}
$$

We start with the first integral in (4.1). Using assumption (1.2), we obtain

$$
\begin{gathered}
\int_{\left\{\left|u-T_{h} v\right| \leq t\right\}} a(x, \nabla u) \cdot \nabla\left(u-T_{h} v\right) d x \geq \int_{E_{1}} a(x, \nabla u) \cdot \nabla(u-v) d x \\
\geq \int_{E_{2}} a(x, \nabla u) \cdot \nabla(u-v) d x-\int_{E_{3}} a(x, \nabla u) \cdot \nabla v d x .
\end{gathered}
$$

By assumption (1.3) and the Hölder inequality (2.1), we estimate the last integral in the above expression as follows:

$$
\begin{aligned}
& \left|\int_{E_{3}} a(x, \nabla u) \cdot \nabla v d x\right| \leq \beta \int_{E_{3}}\left(j(x)+|\nabla u|^{p(x)-1}\right)|\nabla v| d x \\
\leq & 2 \beta\left(\|j\|_{p^{\prime}(\cdot)}+\left\||\nabla u|^{p(x)-1}\right\|_{p^{\prime}(\cdot),\{h<|u| \leq h+t\}}\right)\|\nabla v\|_{p(\cdot),\{h-t<|v| \leq h\}} .
\end{aligned}
$$

The last expression converges to zero as $h$ tends to infinity, by Proposition 3.2 . inequality (2.4), and the following bound for an entropy solution $w$ :

$$
\int_{\{h<|w| \leq h+t\}}|\nabla w|^{p(x)} d x \leq \frac{1}{b} \int_{\{h<|w| \leq h+t\}} a(x, \nabla w) \cdot \nabla w d x \leq \frac{t}{b}\|f\|_{1},
$$

which follows from taking $\varphi=T_{h}(w)$ as a test function in the entropy inequality (1.8). Therefore, from (4.2) and (4.3), we obtain

$$
\int_{\left\{\left|u-T_{h} v\right| \leq t\right\}} a(x, \nabla u) \cdot \nabla\left(u-T_{h} v\right) d x \geq I+\int_{E_{2}} a(x, \nabla u) \cdot \nabla(u-v) d x
$$

where $I$ converges to zero as $h$ tends to infinity. We may adopt the same procedure to treat the second integral in (4.1) and obtain

$$
\int_{\left\{\left|v-T_{h} u\right| \leq t\right\}} a(x, \nabla v) \cdot \nabla\left(v-T_{h} u\right) d x \geq I I-\int_{E_{2}} a(x, \nabla v) \cdot \nabla(u-v) d x
$$

where $I I$ converges to zero as $h$ tends to infinity.

Next, we consider the right-hand side of inequality (4.1). Noting that

$$
T_{t}\left(u-T_{h} v\right)+T_{t}\left(v-T_{h} u\right)=0 \quad \text { in } \quad\{|u| \leq h,|v| \leq h\},
$$

we obtain

$$
\begin{aligned}
& \mid \int_{\Omega} f(x)\left(T_{t}\left(u-T_{h} v\right)+T_{t}\left(v-T_{h} u\right)\right) d x \mid \\
& \leq 2 t\left(\int_{\{|u|>h\}}|f| d x+\int_{\{|v|>h\}}|f| d x\right) .
\end{aligned}
$$

Since both meas $\{|u|>h\}$ and meas $\{|v|>h\}$ tend to zero as $h$ goes to infinity (by Proposition 3.2), the right-hand side of inequality (4.1) tends to zero as $h$ goes to infinity. From this assertion, (4.1), (4.4), and (4.5) we obtain, letting $h \rightarrow+\infty$,

$$
\int_{\{|u-v| \leq t\}}(a(x, \nabla u)-a(x, \nabla v)) \cdot \nabla(u-v) d x \leq 0, \quad \text { for all } t>0 .
$$

By assumption (1.4), we conclude that $\nabla u=\nabla v$, a.e. in $\Omega$. 
Finally, from the Poincaré inequality (2.2), we have

$$
\left\|T_{t}(u-v)\right\|_{p(\cdot)} \leq C\left\|\nabla\left(T_{t}(u-v)\right)\right\|_{p(\cdot)}=0, \quad \text { for all } t>0,
$$

and hence $u=v$, a.e. in $\Omega$.

\section{Existence of ENTROPy AND WEAK SOlutions}

Let $\left(f_{n}\right)_{n}$ be a sequence of bounded functions, strongly converging to $f \in L^{1}(\Omega)$ and such that

$$
\left\|f_{n}\right\|_{1} \leq\|f\|_{1}, \quad \text { for all } n \text {. }
$$

We consider the problem

$$
\left\{\begin{aligned}
-\operatorname{div}(a(x, \nabla u)) & =f_{n}(x) & & \text { in } \quad \Omega \\
u & =0 & & \text { on } \quad \partial \Omega .
\end{aligned}\right.
$$

It follows from a standard modification of the arguments in [16, Theorem 4.2] that problem (5.2) has a unique weak energy solution $u_{n} \in W_{0}^{1, p(\cdot)}(\Omega)$. Our aim is to prove that these approximate solutions $u_{n}$ tend, as $n$ goes to infinity, to a measurable function $u$ which is an entropy solution of the limit problem (1.1). We will divide the proof into several steps and use as the main tool the a priori estimates for $u_{n}$ and its gradient obtained in section 3 Much of the reasoning is based on the ideas developed in [7, [5, and [3]; although some of the arguments are not new, we have decided to present a self-contained proof for the sake of clarity and readability.

We start by proving that the sequence $\left(u_{n}\right)_{n}$ of solutions of problem (5.2) converges in measure to a measurable function $u$.

Proposition 5.1. Assume (1.2)-(1.5), $f \in L^{1}(\Omega)$ and (5.1). Let $u_{n} \in W_{0}^{1, p(\cdot)}(\Omega)$ be the solution of (5.2). The sequence $\left(u_{n}\right)_{n}$ is Cauchy in measure. In particular, there exists a measurable function $u$ such that $u_{n} \rightarrow u$ in measure.

Proof. Let $s>0$ and define

$$
E_{1}:=\left\{\left|u_{n}\right|>t\right\}, E_{2}:=\left\{\left|u_{m}\right|>t\right\} \text {, and } E_{3}:=\left\{\left|T_{t}\left(u_{n}\right)-T_{t}\left(u_{m}\right)\right|>s\right\},
$$

where $t>0$ is to be fixed. We note that

$$
\left\{\left|u_{n}-u_{m}\right|>s\right\} \subset E_{1} \cup E_{2} \cup E_{3},
$$

and hence,

$$
\text { meas }\left\{\left|u_{n}-u_{m}\right|>s\right\} \leq \text { meas }\left(E_{1}\right)+\text { meas }\left(E_{2}\right)+\text { meas }\left(E_{3}\right) \text {. }
$$

Let $\epsilon>0$. Using (5.1) and the uniform bound given by Proposition 3.2, we choose $t=t(\epsilon)$ such that

$$
\text { meas }\left(E_{1}\right) \leq \epsilon / 3 \text { and } \text { meas }\left(E_{2}\right) \leq \epsilon / 3 \text {. }
$$

On the other hand, taking $\varphi=0$ in the entropy condition (1.8) for $u_{n}$ yields

$$
\int_{\Omega}\left|\nabla T_{t}\left(u_{n}\right)\right|^{p(x)} d x \leq \frac{\|f\|_{1}}{b} t, \quad \text { for all } n \geq 0,
$$

using (1.2) and (5.1). Therefore, we can assume, by the Sobolev embedding (2.3), that $\left(T_{t}\left(u_{n}\right)\right)_{n}$ is a Cauchy sequence in $L^{q(\cdot)}(\Omega)$, for all $1 \leq q(\cdot) \ll p^{*}(\cdot)$. Consequently, there exists a measurable function $u$ such that

$$
T_{t}\left(u_{n}\right) \rightarrow T_{t}(u), \quad \text { in } L^{q(\cdot)}(\Omega) \text { and a.e. }
$$


Thus,

$$
\operatorname{meas}\left(E_{3}\right) \leq \int_{\Omega}\left(\frac{\left|T_{t}\left(u_{n}\right)-T_{t}\left(u_{m}\right)\right|}{s}\right)^{q(x)} d x \leq \frac{\epsilon}{3}
$$

for all $n, m \geq n_{0}(s, \epsilon)$.

Finally, from (5.3), (5.4), and the last estimate, we obtain that

$$
\text { meas }\left\{\left|u_{n}-u_{m}\right|>s\right\} \leq \epsilon, \quad \text { for all } n, m \geq n_{0}(s, \epsilon) \text {; }
$$

i.e., $\left(u_{n}\right)_{n}$ is a Cauchy sequence in measure.

In order to prove that the sequence $\left(\nabla u_{n}\right)_{n}$ converges in measure to the weak gradient of $u$ we need the following standard fact in measure theory ( $c f$. [19]).

Lemma 5.2. Let $(X, \mathcal{M}, \mu)$ be a measure space such that $\mu(X)<+\infty$. Consider a measurable function $\gamma: X \rightarrow[0,+\infty]$ such that

$$
\mu(\{x \in X: \gamma(x)=0\})=0 .
$$

Then, for every $\epsilon>0$, there exists $\delta>0$ such that

$$
\mu(A)<\epsilon, \quad \text { for all } A \in \mathcal{M} \text { with } \int_{A} \gamma d \mu<\delta .
$$

Proposition 5.3. Assume (1.2)-(1.5), $f \in L^{1}(\Omega)$ and (5.1). Let $u_{n} \in W_{0}^{1, p(\cdot)}(\Omega)$ be the solution of (5.2). Then $\nabla u_{n}$ converges in measure to the weak gradient of $u$.

Proof. We claim that $\left(\nabla u_{n}\right)_{n}$ is Cauchy in measure. Indeed, let $s>0$, and consider

$$
E_{1}:=\left\{\left|\nabla u_{n}\right|>h\right\} \cup\left\{\left|\nabla u_{m}\right|>h\right\}, \quad E_{2}:=\left\{\left|u_{n}-u_{m}\right|>t\right\},
$$

and

$$
E_{3}:=\left\{\left|\nabla u_{n}\right| \leq h,\left|\nabla u_{m}\right| \leq h,\left|u_{n}-u_{m}\right| \leq t,\left|\nabla u_{n}-\nabla u_{m}\right|>s\right\},
$$

where $h$ and $t$ will be chosen later. We note that

$$
\left\{\left|\nabla u_{n}-\nabla u_{m}\right|>s\right\} \subset E_{1} \cup E_{2} \cup E_{3} .
$$

Let $\epsilon>0$. By Proposition 3.6, we may choose $h=h(\epsilon)$ large enough such that meas $\left(E_{1}\right) \leq \epsilon / 3$ for all $n, m \geq 0$. On the other hand, by Proposition 5.1 (see (5.6)), we have that meas $\left(E_{2}\right) \leq \epsilon / 3$ for all $n, m \geq n_{0}(t, \epsilon)$. Moreover, by assumption (1.4), there exists a real-valued function $\gamma: \Omega \rightarrow[0,+\infty]$ such that $\operatorname{meas}\{x \in \Omega: \gamma(x)=0\}=0$ and

$$
\left(a(x, \xi)-a\left(x, \xi^{\prime}\right)\right) \cdot\left(\xi-\xi^{\prime}\right) \geq \gamma(x),
$$

for all $\xi, \xi^{\prime} \in \mathbb{R}^{N}$ such that $|\xi|,\left|\xi^{\prime}\right| \leq h,\left|\xi-\xi^{\prime}\right| \geq s$, for a.e. $x \in \Omega$ (cf. [7). Let $\delta=\delta(\epsilon)$ be given from Lemma 5.2, replacing $\epsilon$ and $A$ by $\epsilon / 3$ and $E_{3}$, respectively. Using (5.8), the equation, and (5.1), we obtain

$$
\int_{E_{3}} \gamma(x) d x \leq \int_{E_{3}}\left(a\left(x, \nabla u_{n}\right)-a\left(x, \nabla u_{m}\right)\right) \cdot \nabla\left(u_{n}-u_{m}\right) d x \leq 2\|f\|_{1} t<\delta,
$$

choosing $t=\delta /\left(4\|f\|_{1}\right)$. From Lemma [5.2 it follows that meas $\left(E_{3}\right)<\epsilon / 3$. Thus, using (5.7) and the estimates obtained for $E_{1}, E_{2}$, and $E_{3}$, it follows that meas $\left(\left\{\left|\nabla u_{n}-\nabla u_{m}\right| \geq s\right\}\right) \leq \epsilon$, for all $n, m \geq n_{0}(s, \epsilon)$, proving the claim.

As a consequence, $\left(\nabla u_{n}\right)_{n}$ converges in measure to some measurable function $v$. Finally, since $\left(\nabla T_{t} u_{n}\right)_{n}$ is uniformly bounded in $L^{p(\cdot)}(\Omega)$, for all $t>0$, it converges weakly to $\nabla\left(T_{t} u\right)$ in $L^{1}(\Omega)$. Therefore, $v$ coincides with the weak gradient of $u$ (see Proposition 3.1). 
We now prove the main theorem in this paper.

Proof of Theorem 1.2. Fix $t>0, \varphi \in W_{0}^{1, p(\cdot)}(\Omega) \cap L^{\infty}(\Omega)$, and choose $T_{t}\left(u_{n}-\varphi\right)$ as a test function in (1.6), with $u$ replaced by $u_{n}$, to obtain

$$
\int_{\Omega} a\left(x, \nabla u_{n}\right) \cdot \nabla T_{t}\left(u_{n}-\varphi\right) d x=\int_{\Omega} f_{n}(x) T_{t}\left(u_{n}-\varphi\right) d x .
$$

We note that this choice can be made using a standard density argument. We now pass to the limit in the previous identity. Concerning the right-hand side, the convergence is obvious since $f_{n}$ converges strongly in $L^{1}$ to $f$ and $T_{t}\left(u_{n}-\varphi\right)$ converges weakly-* in $L^{\infty}$, and a.e. to $T_{t}(u-\varphi)$.

Next, we write the left-hand side as

$$
\int_{\left\{\left|u_{n}-\varphi\right| \leq t\right\}} a\left(x, \nabla u_{n}\right) \cdot \nabla u_{n} d x-\int_{\left\{\left|u_{n}-\varphi\right| \leq t\right\}} a\left(x, \nabla u_{n}\right) \cdot \nabla \varphi d x
$$

and note that $\left\{\left|u_{n}-\varphi\right| \leq t\right\}$ is a subset of $\left\{\left|u_{n}\right| \leq t+\|\varphi\|_{\infty}\right\}$. Hence, taking $s=t+\|\varphi\|_{\infty}$, we rewrite the second integral in (5.9) as

$$
\int_{\left\{\left|u_{n}-\varphi\right| \leq t\right\}} a\left(x, \nabla T_{s}\left(u_{n}\right)\right) \cdot \nabla \varphi d x .
$$

Since $a\left(x, \nabla T_{s}\left(u_{n}\right)\right)$ is uniformly bounded in $\left(L^{p^{\prime}(\cdot)}(\Omega)\right)^{N}$ (by assumption (1.3) and (5.5) ) and by Proposition 5.3, we have that it converges weakly to $a\left(x, \nabla T_{s}(u)\right)$ in $\left(L^{p^{\prime}(\cdot)}(\Omega)\right)^{N}$. Therefore the last integral converges to

$$
\int_{\{|u-\varphi| \leq t\}} a(x, \nabla u) \cdot \nabla \varphi d x
$$

The first integral in (5.9) is nonnegative, by (1.2), and it converges a.e. by Proposition [5.3. It follows from Fatou's lemma that

$$
\int_{\{|u-\varphi| \leq t\}} a(x, \nabla u) \cdot \nabla u d x \leq \liminf _{n \rightarrow+\infty} \int_{\left\{\left|u_{n}-\varphi\right| \leq t\right\}} a\left(x, \nabla u_{n}\right) \cdot \nabla u_{n} d x .
$$

Gathering results, we obtain

$$
\int_{\Omega} a(x, \nabla u) \cdot \nabla T_{t}(u-\varphi) d x \leq \int_{\Omega} f(x) T_{t}(u-\varphi) d x ;
$$

i.e., $u$ is an entropy solution of (1.1).

The uniqueness follows from Theorem 4.1 and the regularity properties from Corollaries 3.5 and 3.7

To obtain Theorem 1.3 we need to prove, in particular, that $u$ satisfies the equation in the distributional sense, i.e., that (1.6) holds. For this, we need two technical lemmas. The first one is an extension of Lemma 6.1 in [5].

Lemma 5.4. Let $\left(v_{n}\right)_{n}$ be a sequence of measurable functions. If $v_{n}$ converges in measure to $v$ and is uniformly bounded in $L^{q(\cdot)}(\Omega)$, for some $1 \ll q(\cdot) \in L^{\infty}(\Omega)$, then $v_{n} \rightarrow v$ strongly in $L^{1}(\Omega)$. 
Proof. Note first that $L^{q(\cdot)}(\Omega) \subset L^{q-}(\Omega)$, and hence we may assume $\left(v_{n}\right)_{n}$ to be uniformly bounded in $L^{q_{-}}(\Omega)$. Using this fact and the Hölder inequality, we obtain

$$
\begin{aligned}
\int_{\Omega}\left|v_{m}-v_{n}\right| d x & =\int_{\left\{\left|v_{m}-v_{n}\right| \leq s\right\}}\left|v_{m}-v_{n}\right| d x+\int_{\left\{\left|v_{m}-v_{n}\right|>s\right\}}\left|v_{m}-v_{n}\right| d x \\
& \leq|\Omega| s+\operatorname{meas}\left(\left\{\left|v_{m}-v_{n}\right|>s\right\}\right)^{1 / q_{-}^{\prime}}\left\|v_{m}-v_{n}\right\|_{q_{-}} \\
& \leq|\Omega| s+C \operatorname{meas}\left(\left\{\left|v_{m}-v_{n}\right|>s\right\}\right)^{1 / q_{-}^{\prime}},
\end{aligned}
$$

for all $s>0$.

Taking $s$ small enough in (5.10) and using the convergence in measure of $\left(v_{n}\right)_{n}$, we obtain that, for all $\epsilon>0$, there exists $n_{0}=n_{0}(\epsilon)$ such that $\left\|v_{m}-v_{n}\right\|_{1}<\epsilon$, for all $m, n \geq n_{0}(\epsilon)$.

The second technical lemma will be the key point to prove that the entropy solution satisfies the equation in the sense of distributions.

Proposition 5.5. Assume (1.2) $-(1.5), f \in L^{1}(\Omega)$ and (5.1). Let $u_{n} \in W_{0}^{1, p(\cdot)}(\Omega)$ be the solution of (5.2). If $p(\cdot)-1 \ll q_{1}(\cdot)$, then the following assertions hold:

(i) $a\left(x, \nabla u_{n}\right)$ converges to $a(x, \nabla u)$ strongly in $L^{1}(\Omega)$.

(ii) $a(x, \nabla u) \in L^{q(\cdot)}(\Omega)$, for some $1 \ll q(\cdot)$.

(iii) $u$ and $\nabla u$ satisfy (3.4) and (3.6).

Proof. (i) - (ii) By Proposition 5.3 and the Nemitskii Theorem (cf. 24, p. 20]), we obtain that $a\left(x, \nabla u_{n}\right)$ converges to $a(x, \nabla u)$ in measure. Moreover, using (1.3), we have

$$
\left|a\left(x, \nabla u_{n}\right)\right| \leq \beta\left(j(x)+\left|\nabla u_{n}\right|^{p(x)-1}\right),
$$

with $j \in L^{p^{\prime}(\cdot)}(\Omega) \subset L^{q(\cdot)}(\Omega)$, for all $1 \leq q(\cdot) \ll N /(N-1)$. By Corollary 3.7 applied to $u_{n}$, (5.1) and the assumption that $p(\cdot)-1 \ll q_{1}(\cdot)$, we have that $\left(\left|\nabla u_{n}\right|^{p(\cdot)-1}\right)_{n}$ is uniformly bounded in $L^{q(\cdot)}(\Omega)$, for some $1 \ll q(\cdot)$. Hence, using Lemmas 5.3 and 5.4 , we obtain that $a\left(x, \nabla u_{n}\right)$ converges to $a(x, \nabla u)$ strongly in $L^{1}(\Omega)$, and $a(x, \nabla u) \in L^{q(\cdot)}(\Omega)$.

(iii) It follows from taking the limit as $n \rightarrow+\infty$ in Corollaries 3.5 and 3.7 applied to $u_{n}$ and using (5.1).

Proof of Theorem 1.3. Let $u_{n} \in W_{0}^{1, p(\cdot)}(\Omega)$ be the solution of (15.2) and let $u$ be given by Proposition [5.1. Using Proposition 5.5 (i) and the strong convergence in $L^{1}$ of the $f_{n}$ to $f$, we obtain (1.6) by passing to the limit in

$$
\int_{\Omega} a\left(x, \nabla u_{n}\right) \cdot \nabla \varphi d x=\int_{\Omega} f_{n}(x) \varphi d x
$$

for all $\varphi \in C_{0}^{\infty}(\Omega)$.

Now, we claim that $1 \ll q_{1}(\cdot)$ and $1 \ll q_{0}(\cdot)$ under the assumption $2-1 / N \ll p(\cdot)$. By definition,

$$
q_{1}(\cdot)=\frac{p(\cdot)^{2}}{p(\cdot)+p_{+}^{\prime}(1-p(\cdot) / N)} ;
$$

therefore, $1 \ll q_{1}(\cdot)$ if and only if

$$
\frac{p_{+}^{\prime} / p(\cdot)-(p(\cdot)-1)}{p_{+}^{\prime}} \ll \frac{1}{N} .
$$


Noting that the left-hand side is decreasing in $p(\cdot)$ and that $p_{+}^{\prime}=p_{-} /\left(p_{-}-1\right)$, (5.11) is equivalent to

$$
(2-p)_{+}=2-p_{-}=\frac{p_{+}^{\prime} / p_{-}-\left(p_{-}-1\right)}{p_{+}^{\prime}}<\frac{1}{N},
$$

i.e., $(p-2+1 / N)_{-}>0$, proving the first assertion. On the other hand, $1 \ll q_{0}(\cdot)$ is equivalent to

$$
1 \ll\left(p_{-}-1\right) \frac{N p(\cdot)}{p_{-}(N-p(\cdot))} .
$$

Since the right-hand side is increasing in $p(\cdot)$, it is sufficient to prove that

$$
1<\left(p_{-}-1\right) \frac{N}{\left(N-p_{-}\right)},
$$

which follows easily from the assumption $2-1 / N \ll p(\cdot)$.

As a consequence, from Corollaries 3.5 and 3.7 .

$$
u \in L^{q(\cdot)}(\Omega), \quad \text { for all } 1 \leq q(\cdot) \ll q_{0}(\cdot)
$$

and

$$
u \in W_{0}^{1, q(\cdot)}(\Omega), \quad \text { for all } 1 \leq q(\cdot) \ll q_{1}(\cdot) .
$$

Remark 5.6. Assumption $p(\cdot)-1 \ll q_{1}(\cdot)$, which is obviously satisfied for $p$ constant, is equivalent to the condition

$$
\frac{N p^{\prime}(\cdot)}{N-p(\cdot)} \gg p^{\prime} \cdot
$$

The analysis of the behaviour of the function on the left-hand side of this inequality leads to the following conclusions:

(i) if $p_{+}<\sqrt{N}$, then (5.12) is satisfied for any function $p(\cdot)$ such that

$$
\frac{1}{p_{-}}-\frac{1}{p_{+}}<\frac{p_{+}-1}{N}
$$

(ii) if $p_{-} \leq \sqrt{N} \leq p_{+}$, then (5.12) is satisfied for any function $p(\cdot)$ such that

$$
p_{-}>\frac{N}{2 \sqrt{N}-1}
$$

(iii) if $p_{-}>\sqrt{N}$, then (5.12) is satisfied for any function $p(\cdot)$.

The condition in case (i) only holds if $p_{-}$is close to $p_{+}$, so it forces a modest variation in the field of values of $p(\cdot)$.

Remark 5.7. We finally comment on the significance of assumption $p(\cdot)-1 \ll q_{1}(\cdot)$, under $2-1 / N \ll p(\cdot)$. Observe that:

- It always holds if $p(\cdot) \ll 2$ since then $p(\cdot)-1 \ll 1 \ll q_{1}(\cdot)$.

- The condition in case (i) above is only pertinent for dimensions $N \geq 5$ since $p(\cdot) \ll 2$ if $p_{+}<\sqrt{N}$, for $N=2,3,4$. Moreover, for $N \geq 5$, the condition is satisfied if

$$
\sup _{p_{-}>2-\frac{1}{N}} \frac{1}{p_{-}}=\frac{N}{2 N-1}<\frac{2 \sqrt{N}-1}{N}=\inf _{2 \leq p_{+}<\sqrt{N}}\left\{\frac{1}{p_{+}}+\frac{p_{+}-1}{N}\right\},
$$

which holds for $N \leq 10$.

- The condition in case (ii) above always holds for dimensions $N \leq 10$, since then $\frac{N}{2 \sqrt{N}-1}<2-\frac{1}{N}$. 
Therefore, up to dimension $N=10$, assumption $p(\cdot)-1 \ll q_{1}(\cdot)$ is automatically satisfied when we assume $2-1 / N \ll p(\cdot)$.

\section{ACKNOWLEDGEMENTS}

In an earlier version of this paper, use was made of an invalid Poincaré type inequality, stated and (erroneously) proved in [Y.Q. Fu, The existence of solutions for elliptic systems with nonuniform growth, Studia Math. 151 (2002), 227-246]. We thank Professor Fumi-Yuki Maeda, from Hiroshima, for bringing this fact to our attention and for providing a counterexample to the false assertion.

\section{REFERENCES}

[1] E. Acerbi and G. Mingione, Regularity results for a class of functionals with nonstandard growth, Arch. Ration. Mech. Anal. 156 (2001), 121-140. MR1814973 (2002h:49056)

[2] E. Acerbi and G. Mingione, Gradient estimates for the $p(x)$-Laplacian system, J. reine angew. Math. 584 (2005), 117-148. MR2155087 (2006f:35068)

[3] A. Alvino, L. Boccardo, V. Ferone, L. Orsina and G. Trombetti, Existence results for nonlinear elliptic equations with degenerate coercivity, Ann. Mat. Pura Appl. 182 (2003), 53-79. MR.1970464 (2004e:35054)

[4] S. Antontsev and S. Shmarev, A model porous medium equation with variable exponent of nonlinearity: Existence, uniqueness and localization properties of solutions, Nonlinear Anal. 60 (2005), 515-545. MR2103951(2005m:35140)

[5] Ph. Bénilan, L. Boccardo, T. Gallouët, R. Gariepy, M. Pierre and J.L. Vázquez, An L $L^{1}$-theory of existence and uniqueness of solutions of nonlinear elliptic equations, Ann. Sc. Norm. Super. Pisa, Cl. Sci. 22 (1995), 241-273. MR1354907 (96k:35052)

[6] L. Boccardo and T. Gallouët, Nonlinear elliptic and parabolic equations involving measure data, J. Funct. Anal. 87 (1989), 149-169. MR1025884 (92d:35286)

[7] L. Boccardo and T. Gallouët, Nonlinear elliptic equations with right-hand side measures, Comm. Partial Differential Equations 17 (1992), 641-655. MR.1163440 (94c:35083)

[8] Y. Chen, S. Levine and M. Rao, Variable exponent, linear growth functionals in image restoration, SIAM J. Appl. Math. 66 (2006), 1383-1406. MR.2246061 (2007d:94004)

[9] M. Crandall and P. Rabinowitz, Some continuation and variational methods for positive solutions of nonlinear elliptic eigenvalue problems, Arch. Ration. Mech. Anal. 58 (1975), 207-218. MR0382848 (52:3730)

[10] E. DiBenedetto, $C^{1+\alpha}$ local regularity of weak solutions of degenerate elliptic equations, Nonlinear Anal. 7 (1983), 827-850. MR709038 (85d:35037)

[11] E. DiBenedetto and J. Manfredi, On the higher integrability of the gradient of weak solutions of certain degenerate elliptic systems, Amer. J. Math. 115 (1993), 1107-1134. MR1246185 (94i:35077)

[12] E. DiBenedetto, J.M. Urbano and V. Vespri, Current issues on singular and degenerate evolution equations, in: Handbook of Differential Equations, Evolutionary Equations, vol. 1, pp. 169-286, Elsevier, 2004. MR.2103698 (2006b:35160)

[13] L. Diening, Riesz potential and Sobolev embeddings on generalized Lebesgue and Sobolev spaces $L^{p(\cdot)}$ and $W^{k, p(\cdot)}$, Math. Nachr. 268 (2004), 31-43. MR2054530 (2005d:46071)

[14] L. Diening, P. Hästö and A. Nekvinda, Open problems in variable exponent Lebesgue and Sobolev spaces, in: FSDONA04 Proceedings, Drabek and Rakosnik (eds.), pp. 38-58, Milovy, Czech Republic, 2004.

[15] D. Edmunds and J. Rákosník, Sobolev embeddings with variable exponent, Studia Math. 143 (2000), 267-293. MR1815935 (2001m:46072)

[16] X. Fan and Q. Zhang, Existence of solutions for $p(x)$-Laplacian Dirichlet problem, Nonlinear Anal. 52 (2003), 1843-1852. MR1954585 (2004f:35060)

[17] X. Fan and D. Zhao, A class of De Giorgi type and Hölder continuity, Nonlinear Anal. 36 (1999), 295-318. MR.1688232 (2000a:49072)

[18] J. Fleckinger, E. Harrell and F. de Thélin, Boundary behavior and estimates for solutions of equations containing the p-Laplacian, Electron. J. Differential Equations 1999 (1999), 1-19. MR.1713597 (2000k:35083) 
[19] P. Halmos, Measure theory, D.Van Nostrand Company, New York, 1950. MR0033869 $(11: 504 \mathrm{~d})$

[20] P. Harjulehto, P. Hästö, M. Koskenoja and S. Varonen, The Dirichlet energy integral and variable exponent Sobolev spaces with zero boundary values, Potential Anal. 25 (2006), 205222. MR 2255345 (2008f:35119)

[21] E. Henriques and J.M. Urbano, Intrinsic scaling for PDEs with an exponential nonlinearity, Indiana Univ. Math. J. 55 (2006), 1701-1722. MR2270934 (2007h:35164)

[22] J. Kinnunen and S. Zhou, A boundary estimate for nonlinear equations with discontinuous coefficients, Differential Integral Equations 14 (2001), 475-492. MR.1799417(2002a:35070)

[23] O. Kováčik and J. Rákosník, On spaces $L^{p(x)}$ and $W^{1, p(x)}$, Czechoslovak Math. J. 41 (1991), 592-618. MR1134951 (92m:46047)

[24] M. Krasnosel'skii, Topological methods in the theory of nonlinear integral equations, Pergamon Press, New York, 1964. MR0159197 (28:2414)

[25] A. Kufner, J. Oldřich and S. Fučík, Function spaces, Noordhoff International Publishing, Leyden, 1977. MR 0482102 (58:2189)

[26] J. Leray and J.L. Lions, Quelques résultats de Višik sur les problèmes elliptiques non linéaires par les méthodes de Minty-Browder, Bull. Soc. Math. France 93 (1965), 97-107. MR0194733 (33:2939)

[27] P. Marcellini, Regularity of minimizers of integrals of the calculus of variations with nonstandard growth conditions, Arch. Ration. Mech. Anal. 105 (1989), 267-284. MR969900 (90a:49017)

[28] M. Růžička, Electrorheological fluids: Modeling and mathematical theory, Lecture Notes in Mathematics 1748, Springer-Verlag, Berlin, 2000. MR1810360(2002a:76004)

[29] I. Sharapudinov, On the topology of the space $L^{p(t)}([0 ; 1])$, Math. Notes 26 (1979), 796-806. [Translation of Mat. Zametki 26 (1978), 613-632.] MR552723(81c:46021)

[30] P. Tolksdorf, Regularity for a more general class of quasilinear elliptic equations, J. Differential Equations 51 (1984), 126-150. MR727034 (85g:35047)

[31] V. Zhikov, Averaging of functionals of the calculus of variations and elasticity theory, Izv. Akad. Nauk SSSR Ser. Mat. 50 (1986), 675-710, 877. MR864171(88a:49026)

CMuC, Department of Mathematics, University of Coimbra, 3001-454 Coimbra, PorTUGAL

E-mail address: msanchon@mat.uc.pt

Current address: Departament de Matemàtica Aplicada i Anàlisi, Facultat de Matemàtiques, Universitat de Barcelona, Gran Via 585, E-08007 Barcelona, Spain

CMuC, Department of Mathematics, University of Coimbra, 3001-454 Coimbra, PorTUGAL

E-mail address: jmurb@mat.uc.pt 